\title{
Improving acute medical management: Junior Doctor Emergency Prescription Cards
}

\author{
Joe Hutton, Megan Gingell, Lisa Hutchinson \\ Gloucestershire NHS
}

\begin{abstract}
Doctors commencing Foundation Year (FY) training face many stresses and challenges. FY doctors are often the first point of contact for acutely unwell and deteriorating patients. Trust guidelines are used to aid acute medical management. Accessing guidelines is often fraught with barriers. Evidence suggests aide-memoire cards can provide easier access to guidelines and management pathways.
\end{abstract}

We aimed to improve prescribing accuracy and efficiency of FY doctors for acute medical conditions within Gloucestershire trust by improving access to and usability of trust guidelines.

Questionnaires were distributed to FY doctors to identify acute medical conditions to include on the emergency prescription cards (EPCs). Two small double-sided cards were created containing bullet pointed trust guidelines for: hyper/hypokalaemia, status epilepticus, diabetic emergencies, arrhythmias, myocardial infarction, acute asthma, pulmonary oedema, anaphylaxis and a ward-round checklist. Feedback was used to improve EPCs prior to distribution. Pre $(\mathrm{N}=53)$ and post-intervention $(\mathrm{N}=46)$ written questionnaires were completed by $\mathrm{FY}$ doctors. These assessed acute clinical management including use of guidance, confidence in management, speed of prescribing and EPC "usability". To assess prescribing accuracy, prescriptions for acute medical conditions were reviewed pre $(\mathrm{N}=8)$ and post-intervention $(\mathrm{N}=12)$.

The EPCs were well received (80\% quite/very useful) and found "easy to use" (83\%). The introduction of EPCs increased guidance use (preintervention $58.8 \%$, post-intervention $71.7 \%$ ), increased confidence (pre-intervention $79 \%$, post-intervention $89 \%$ ) and significantly improved prescribing speed $(p=0.05)$. There was a significant correlation with confidence and prescribing speed $(p=0.023)$. The accuracy of prescribed doses improved (pre-intervention $62.5 \%$, post-intervention $87.5 \%$ accurate) as did details regarding route / additional required information (preintervention $75 \%$, post-intervention $97.7 \%$ ).

The EPCs support the management of unwell patients, are relevant to the workload of modern doctors practice and may improve patient care. This improvement measure could be applied to other NHS trusts and medical specialties.

\section{Problem}

Life as a doctor involves managing acutely unwell patients. A major stressor for new doctors is becoming the first point of contact for an unwell patient (1-4). Despite initiatives to ease this transition, many newly qualified doctors feel uneasy with this dramatic switch $(1,3)$.

Individual Trusts can make different recommendations regarding preferred treatment regimens. E-guidance is frequently used but research suggests that there are often difficulties in accessing guidance (5). These barriers include the availability of computers, internet-access and "signposting" of the required piece of guidance on online systems.

Error in medicine has been well-documented for many years $(6,7)$. Despite safety strategies, episodes of sub-optimal care and adverse incidents occur (6-8). Difficulties in accessing guidance create scope for error and treatment delays (5). Some support for this is found in paediatric practice (5) and is likely to apply equally to adult medicine. Safety data suggests that variability in methodology exacerbates errors, both in primary and secondary care (9-11). Uniformity in practice is well-documented to reduce potential for errors (9). Ready access to guidance should help reduce variability and inappropriate prescribing, enhancing patient safety and reducing hospital spending.

Gloucestershire NHS Trust consists of two busy district general hospitals, which together manage over 40,000 admissions per year (12). As part of the wider Severn deanery, it has a large number of incoming Foundation year (FY) doctors. The majority of these have not worked in the Severn area previously. This includes many Foundation Year 2 doctors who change Trust between Foundation Years. As such, it is perfectly placed for assessing this problem and instituting change.

The overall aim of the project was to improve the safety and efficacy of prescribing for acutely unwell patients to improve patient outcome. It also aimed to boost doctors' confidence with managing patients. A secondary aim was to improve doctors' ease of access to and use of hospital guidelines for acutely unwell patients.

\section{Background}

Different strategies have been implemented at a national level to 
ease this difficult transition. Indeed, "Tomorrow's Doctors"” outline the requirement for and benefits of workplace based shadowing (13). Utilising simulation training and providing adequate workplace induction also support newly qualified doctors (13). Despite this, research suggests continued strategies are necessary to facilitate safe and effective progression through training $(1,3)$.

A potential strategy is to aid $\mathrm{FY}$ doctors in accessing point-of-care guidance such as aide-memoire cards. Use of guidance in this manner has the benefit of working to reduce some of the inherent variability in clinical practice. Computerised aide-memoire tools have been previously used for drug dose reminders (15). Other institutions have trialled the use of cards for various healthcare problems with varying degrees of success $(16,17)$. This suggests that a point-of-care system like aide-memoire cards for acute medical emergencies has capacity to enhance doctors' confidence and practice.

\section{Baseline measurement}

Initial data was collected in Gloucestershire NHS Trust (Cheltenham General Hospital and Gloucester Royal Hospital). The pharmacy teams based in the acute admissions units at both sites carried out a pre-intervention audit over a two-week period in November 2013. Three common conditions were looked at: atrial fibrillation with fast ventricular response, pulmonary oedema and hyperkalaemia. Prescriptions for these conditions were assessed as to whether they followed Trust-recommended guidance and whether the correct dose, route and additional instructions had been correctly completed. 8 prescriptions were analysed. In $62.5 \%$, the correct dose was prescribed. In $87.5 \%$, the correct route/additional instructions were used, highlighting a potential for improvement

Simultaneously, data was collected from FY doctors via a written questionnaire between August 2013 to November 2013. This assessed their perceived management of a recent acutely unwell patient. 53 responses were analysed. 58.8\% used guidance to aid the management of their acutely unwell patient, $80 \%$ of which was Trust guidance. The doctor was also asked to estimate their time taken to manage the patient and the confidence they felt. The nature of the acute emergency was also documented.

There was a strong association ( $P=0.095$, Fisher's exact test) between time to institute management and confidence with treatment plan. This indicates that improvements in confidence may facilitate faster management for unwell patients.

See supplementary file: ds7130.pdf - "appendix 1 14.02.16"

\section{Design}

Discussions were held between FY doctors to identify which acute medical problems they would like included on rapid-guidance cards. This included discussion around inclusion of antimicrobial guidance. Upon the strong recommendation of pharmacists and microbiologists, this was not included. This was due to more frequent changes in practice than other areas.

The idea of rapid-guidance cards received a positive response from the cohort. Subsequently, emergency prescription cards (EPCs) were created. These were two double-sided credit-card sized documents (Appendix 1). These listed the Trust guidelines for the management of:

- Hyper/hypokalaemia

- Status epilepticus

- Diabetic emergencies including diabetic ketoacidosis and hyperosmolar hyperglycaemic state

- Tachyarrhythmias and bradyarrhythmias

- Myocardial infarction

- Acute asthma exacerbations

- Pulmonary oedema

- Anaphylaxis

- Ward-round checklist

The chosen design was deemed the most logical intervention for rapid-access portable guidance. The content and formatting was created using multiple rounds of feedback. Each condition was colour coded according to speciality sub-type and formatted for ease of use. The accuracy of contained information was verified by both clinical pharmacists and senior clinicians. The completed cards were submitted to the Trust board for approval.

Upon approval, the EPCs were printed through a well- known national printing company at a cost of around $£ 20$ for 250 cards and was reimbursed through allocated funding from the local quality improvement initiative. EPCs were distributed at Foundation doctor teaching.

Sustainability was considered. To ensure continued relevance, the lead pharmacist agreed to update the content as required. Future printing costs were also reduced by providing the guidance in PDF format for smartphones.

\section{Strategy}

A draft version of the EPCs were created and reviewed by the junior doctor forum, senior clinicians, pharmacy team and Trust approval board.

Creation of EPCs were planned after identifying the problem. Extensive discussions were held with FY doctors to establish which conditions were to be included as previously mentioned. These recommendations were then correlated with the findings from the baseline measurement of commonly encountered acute medical problems. This was to ensure the topics included were relevant and useful. The EPCs were then produced based on this initial feedback.

The draft EPCs were informally introduced to a small cohort of FY doctors on both acute medical and surgical wards. These were used as part of their daily workload, including on-call days when responsible for ward-cover. Reviewers were invited to provide feedback on their appearance, usability, organisation of information 
and content. This highlighted several areas for improvement. Based on this feedback, acute emergencies were colour-coded according to their corresponding clinical specialty. The information in each section was also reformatted to increase usability.

Edited EPCs were re-introduced and reviewed over a two-week period by junior medical staff, medical registrars, clinical pharmacists and consultants. Further feedback was used to guide subsequent improvements. One respondent suggested that the document should be made available in PDF format for use on smartphones. Minor changes to content were made. These included the addition of criteria for discussion with intensive care in the case of diabetic ketoacidosis as well as some extraneous detail for hyperosmolar hyperglycaemic state being removed. A section to support junior-led ward rounds with a checklist of things to check was also added. Several rounds of re-introduction and alterations were made over several months in total.

The baseline results and card development, format and reception was presented at the End of Year Quality Improvement meeting. The final version of the EPCs (appendix 1) were submitted to and approved by the Trust's safety and guidance committee. The EPCs were printed and distributed in August 2014. Electronic versions of EPCs were made available for use on smartphones and uploaded to the front page of the Treatment Guidance page on the Trust's Intranet. The EPCs were publicised widely and disseminated to current Foundation year doctors via mandatory Foundation teaching sessions, word of mouth, email and social media. Doctors were allowed free usage of the cards for several months. Repeat pharmacy data was prospectively obtained over a two-week period in November 2014 using the same data-collection tool as before. Repeat assessment questionnaires were sent to $\mathrm{FY}$ doctors over a two-month period between September to November 2014. This data was analysed and compared to the pre-intervention (baseline measurement) results. The results of the EPCs were presented at the Trust's Quality improvement day in July 2015 in addition to several national conferences.

\section{Results}

The two main outcome measurements were safety of prescription and efficiency of management. The safety of prescriptions was measured by comparing the post-intervention pharmacy audit for the three common clinical emergencies to the baseline measurement audit. Management efficiency was measured via comparison of the questionnaire responses pre and post EPCs introduction. Secondary measures of safety included the use and nature of guidance and the confidence doctors felt with their management plan.

The data collected was analysed for statistical significance using Fisher's exact test. The data set included 53 responses preintervention and 46 post-intervention for the questionnaire data. The pharmacy audit contained 8 responses pre-intervention and 12 postintervention.

It was demonstrated that the EPCs cover the majority (67.9\%) of clinical emergencies encountered by FY doctors. The majority of the emergencies not covered by the cards were caused by sepsis $(60.5 \%)$. A small minority of the remaining emergencies not covered included type 2 respiratory failure, haemorrhage and acute stroke. The content of the EPCs is therefore valid for the common workload of a FY doctor. As previously mentioned, the decision not to include antibiotic guidance had been made due to multi-source advice and the Trust already widely uses comprehensive 'Sepsis Six' care bundles.

The EPCs increased the proportion of doctors checking guidance (71.7\%, pre-intervention $58.8 \%$, table 1 ). More doctors checked Trust guidance $(87.8 \%$, pre-intervention $80 \%$, table 1). Additionally, there was an improvement in the confidence of doctors with their management plan (89\%, 79\% pre-intervention, table 1). The EPCs also improved the speed in which management plans were initiated (figure $1, P=0.05$ ). Confidence was demonstrated to be associated with the speed of prescription ( $P=0.095$, Fisher's exact test).

The EPCs were well-received by FY doctors. The majority found them "quite easy" (48\%) or "very easy" (38\%) to use. Additionally, $61 \%$ of FY doctors found them "quite useful" and $19.6 \%$ found them "very useful".

The EPCs led to an apparent increase in the correct dose of prescriptions $(75 \%, 62.5 \%$ baseline). The accuracy of route / additional instructions also apparently increased $(91.7 \%$, baseline $87.5 \%)$. However, neither measure was statistically significant.

See supplementary file: ds7304.pdf - "table 1 and figure 1 11.03.16"

\section{Lessons and limitations}

Introduction of the EPCs seemingly improved doctors' use of guidance and the speed and confidence in which they initiate a management plan for an unwell patient. The EPCs cover a large proportion of the commonly encountered emergencies facing FY doctors on a routine ward shift. There was also an observed improvement in the accuracy of prescriptions observed by ward pharmacists although this did not reach statistical significance. This demonstrates that EPCs have a role in improving doctors' practice, confidence and patient safety.

However, one critical limitation of this study was the use of only two formal data collection periods. This was due to logistical reasons. To ensure sufficient sized samples, a lengthy time frame was required. When combined with the additional pressures of rotating FY doctors and the need to assess doctors at a similar stage in training, only two formal large periods of data collection were included. Pharmacy data sets were also of relatively small size. This was due to similar reasons - only a small proportion of patients developed these conditions during the study period and constant data collection is very labour intensive for the pharmacy team. The pharmacy data was also obtained only from the acute medical wards and thus patients with these conditions in other wards may have been missed. In future data gathering, a longer period of datagathering should be considered with more hospital wards included.

Despite this, efforts were made to ensure that this demonstrates 
sustainable change. Statistical measures were used to compare outcomes. The period between introduction of the cards and data collection was deliberate to try and minimise any temporary shortlived effect.

Much of the observed change demonstrates subjective improvement in behaviour. The apparent improvement in prescribing data suggests the potential for some objective improvement in behavioural practices. This could be better delineated with larger and more regular pharmacy data collection.

Following further feedback and wider use, opportunities for future editions have been highlighted. These include: increasing durability of cards (via lamination or printing on plastic), provision of a larger version with larger font size and inclusion of guidance on additional emergencies such as acute stroke, major haemorrhage and the 'sepsis six.

Given the unexpected uptake of the EPCs amongst allied healthcare professionals such as Advanced Nurse Practitioners, further interventions to increase access of the EPCs to higher-level trainees and locum doctors is being considered.

All groups surveyed expressed desire for a smartphone application covering the same information. This would circumvent printing costs, issues with durability and potentially widen the scope of the project to allow access to antibiotic and more detailed guidance. This would ensure the EPCs stay more sustainable and relevant in the long term. To achieve this, the lead clinical pharmacist has agreed to work with incoming doctors to ensure that the EPCs remain up-to-date and relevant. Planned repeat data collection will ensure further assessment of the sustainability of changes demonstrated.

\section{Conclusion}

This quality-improvement project suggests a role for EPCs in supporting doctors in the transition from medical school to practicing physician. Improving confidence in this manner seems to improve the time-frames in which junior doctors institute initial management for unwell patients. The cards are easy to read, useful and relevant to the workload of modern junior doctors. In addition, the cards may have a role in supporting safe and cost-effective prescribing for unwell patients.

Continued development of this project will ensure the ongoing utility and relevance of the EPCs. There is still future work required in assessing the impact on prescribing accuracy and efficiency. Despite these limitations, EPCs seem to boost doctors' confidence with unwell patients in this period of difficult transition and promote patient safety.

\section{References}

1. Berridge EJ; Freeth D; Sharpe J; Roberts CM. Bridging the gap: supporting the transition from medical student to practicing doctor-a two-week preparation programme after graduation. Medical Teacher 2007; 29 (2-3): 119-127.

2. Cameron A; Millar J; Szmidt N; Hanlon K; Cleland J. Can new doctors be prepared for practice? A review. The clinical teacher 2014; 11(3):188-92.

3. Brennan N.; Corrigan O.; Allard J.; Archer J.; Barnes R.; Bleakley A.; Collett T.; de Bere SR. The transition from medical student to junior doctor: today's experiences of Tomorrow's Doctors. Medical Education 2010; 44(5):449-58.

4. Prince $\mathrm{K}$, van de Wiel M, van der Vleuten $\mathrm{C}$, Boshuizen $\mathrm{H}$; Scherpbier A. Junior doctors' opinions about the transition from medical school to clinical practice: A change of environment. Education for Health 2004; 17(3):323-31.

5. An audit of adherence to guideline based post-operative paediatric prescriptions in a district general hospital. Regional Anesthesia and Pain Medicine 2012: 37(5) supplement 1 E301: 1098-7339.

6. Lucian L, Leape MD. Error in Medicine. The Journal of the American Medical Association 1994; 272(23): 1851-1857.

7. Dorman T, Ashcroft D, Heathfield H, Lewis P, Miles J, Taylor $\mathrm{D}$, Tully $\mathrm{M}$, Wass $\mathrm{V}$. An in depth investigation in causes of prescribing errors by foundation trainees in relation to their medical education. EQUIP study. General Medical Council 2009.

8. Morrow D, North R. Reducing and mitigating human error in Medicine. Reviews of Human Factors and Ergonomics 2005; 1(1): 254-296.

9. Jong J, Groenewegen P, Spreeuwenberg P, Schellevis F, Westert G. Do guidelines create uniformity in medical practice? Social Science \& Medicine 2010: 70(2): 209-216.

10. Grilli R, Magrini N, Penna A, Mura G, Liberati A. Practice guidelines developed by speciality societies: the need for a critical appraisal. The Lancet 2000; 355 (9188): 103-106.

11. Hutchinson A, McIntosh A, Cox S, Gilbert C. Towards efficient guidelines: how to monitor guideline use in primary care. Health Technol Assess 2003; 7 (18):1-97.

12. Gloucestershire Hospitals NHS Foundation Trust. Emergency Pathway Monthly Report [internet]. 2014 [cited 2015 July] Available from URL:

http://www.gloshospitals.nhs.uk/SharePoint2/Board\%20Pap ers/2014/July\%202014/Item\%2012\%20-\%20Emx\%20Pathw ay\%20Monthly\%20Report\%20-\%20Complete.pdf

13. General Medical Council. Tomorrow's Doctors: Outcomes and standards for undergraduate medical education [internet] 2009 [cited 2015 July] Available at URL: http://www.gmcuk.org/Tomorrow s Doctors 1214.pdf 48905759.pdf

14. Okuda Y, Bryson E, DeMaria S, Jacobson L, Quinones J, Shen B, Levine A. , The Utility of Simulation in Medical Education: What Is the Evidence? Mt Sinai J Med 2009; 76 (4) 330-343.

15. Sard B, Walsh K, Doros G, Hannon M, Moschetti W, Bauchner $\mathrm{H}$. Retrospective evaluation of a computerized physician order entry adaptation to prevent prescribing errors in a pediatric emergency department. Paediatrics 2008; 122 (4): 782-786.

16. McCallum Z, South M. Development and use of a portable paediatric resuscitation card. Journal of Paediatrics and Child Health 2004; 40(8): 477-480. 
17. Lenz H, Pascual R, Miller D, Haponik E. Evaluating resident learning behavior in the medical ICU with distribution of a pocket reference card and confidence survey. American Thoracic Society International Conference 2010.

\section{Declaration of interests}

None declared

\section{Acknowledgements}

We would like to thank Dr Marie Wheeler, Pamela Adams and Alistair Graham for all their help.

\section{Ethical approval}

Ethical approval was not sought as it did not qualify as necessary according to local guidelines. 